\title{
COVID-19 and heart damage
}

\section{Editorial}

The link between the SARS-CoV-2 coronavirus and the occurrence of heart problems was discovered almost immediately after the pandemic began. However, for a long time, scientists were unable to establish whether the virus had a direct effect or whether the heart abnormalities were due to an inflammatory response.

Chinese experts were among the first to talk about the likely heart complications after COVID-19. In March, they examined 416 patients with confirmed coronavirus and found serious damage to the heart muscle in about 20 per cent. They also cited data that in the intensive care unit of a Wuhan hospital, arrhythmia was diagnosed in 44 percent of patients. The heart disturbances are the result of a cytokine storm, a potentially dangerous reaction of the body that destroys tissue at the site of inflammation. Myocarditis and arrhythmia, in particular, were the result. ${ }^{1}$

The damage to the heart can be long-lasting. Even two and a half months after recovery, 78 percent of patients have abnormal heart function. Sixty percent show signs of myocardial inflammation. The extent of the damage does not depend on how severe the illness was. Serious complications have occurred even in those who have had COVID-19 asymptomatically. ${ }^{2}$

The virus does not just cause inflammation in the heart muscle, but also infects its cells. Traces of SARS-CoV-2 have been detected in the heart tissue of more than 60 per cent of those who have died of COVID-19. And sixteen people recorded clinically significant levels of viral load in the heart muscle at the time of death. ${ }^{3}$ Damage to the heart in patients with COVID-19 is caused by the virus entering and replicating inside the cells of the heart muscle, causing them to die and disturbing the rhythm of the contractions. ${ }^{4}$

Using stem cells to build human heart tissue, the researchers conducted an experiment and found that the viral infection not only kills the cells but also destroys the muscle fibres responsible for heart muscle contraction. This process can occur even in the absence of an inflammatory response in the body.

At the start of the pandemic, it was found that this coronavirus could cause heart failure or heart damage in otherwise healthy people. Even some athletes who recovered and returned to standard training later developed heart scarring. Patients with COVID-19 who developed heart failure had severe damage to the heart muscle cells, and inflammation is not the original cause of this damage. ${ }^{4}$ Other viral infections also adversely affect the heart in some cases, but COVID-19 induces a different immune response in the heart compared to them, but why this happens remains unclear.

Almost half of people who have had COVID-19 have serious heart problems. The most common are myocarditis, arrhythmias, acute and chronic heart failure, and limited cardiomyocyte necrosis. Serious complications also occur in asymptomatic infected
Volume I4 Issue 4 - 202 I

\author{
Pavel F Zabrodsky \\ Department of Toxicology, Saratov Medical University “REAVIZ, \\ Russia
}

Correspondence: Pavel F Zabrodsky, Saratov Medical University “REAVIZ”, Saratov, Russia, Tel +7 90532327 5I, Email pfzabrodsky@gmail.com

Received: July 18, 2021 | Published: August 23, 2021

persons. Considering that $30-40$ per cent of those infected have no manifestations of the disease, there will be many more cardiac abnormalities associated with COVID-19 than are currently reported. ${ }^{5}$

Complications depend not only on the severity of the infection, but also on many other factors: age, comorbidities, genetic predisposition.

\section{Acknowledgments}

None.

\section{Conflicts of interest}

Author declares there are no conflicts of interest.

Funding

None.

\section{References}

1. Shaobo Shi, Mu Qin, Bo Shen, et al. Association of Cardiac Injury with Mortality in Hospitalized Patients with COVID-19 in Wuhan, China. JAMA Cardiol. 2020;5(7):802-810.

2. Puntmann VO, Carerj L, Wieters I. Outcomes of Cardiovascular Magnetic Resonance Imaging in Patients Recently Recovered From Coronavirus Disease 2019 (COVID-19). JAMA Cardiol. 2020;5(11):1265-1273.

3. Lindner D, Fitzek A, Bräuninger H, et al. Association of Cardiac Infection with SARS-CoV-2 in Confirmed COVID-19 Autopsy Cases. JAMA Cardiol. 2020;5(11):1281-1285.

4. Bailey AL, Dmytrenko O, Greenberg L, et al. SARS-CoV-2 Infects Human Engineered Heart Tissues and Models COVID-19 Myocarditis. JACC Basic Transl Sci. 2021;6(4):331-345.

5. Eric $J$ Topol. COVID-19 can affect the heart. Science. 2020:370(6515):408-409. 